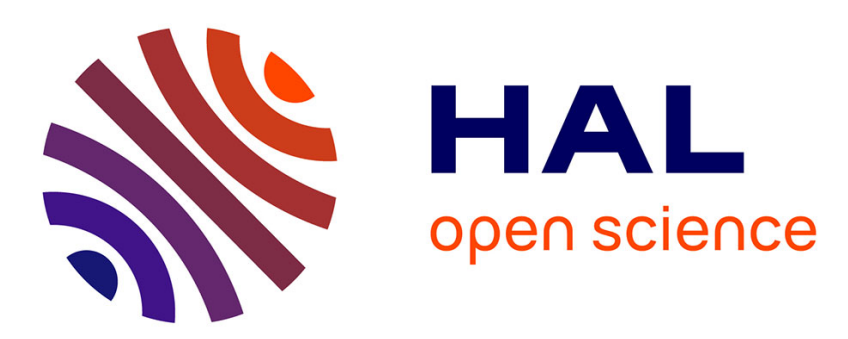

\title{
A Relational Database Integrity Framework for Access Control Policies
}

\author{
Romuald Thion, Stéphane Coulondre
}

\section{To cite this version:}

Romuald Thion, Stéphane Coulondre. A Relational Database Integrity Framework for Access Control Policies. Journal of Intelligent Information Systems, 2012, 38 (1), pp.131-159. 10.1007/s10844-0100146-z . hal-01352933

\section{HAL Id: hal-01352933 https://hal.science/hal-01352933}

Submitted on 7 Mar 2017

HAL is a multi-disciplinary open access archive for the deposit and dissemination of scientific research documents, whether they are published or not. The documents may come from teaching and research institutions in France or abroad, or from public or private research centers.
L'archive ouverte pluridisciplinaire HAL, est destinée au dépôt et à la diffusion de documents scientifiques de niveau recherche, publiés ou non, émanant des établissements d'enseignement et de recherche français ou étrangers, des laboratoires publics ou privés. 


\title{
A Relational Database Integrity Framework for Access Control Policies
}

\author{
Romuald Thion · Stéphane Coulondre
}

the date of receipt and acceptance should be inserted later

\begin{abstract}
Access control is one of the most common and versatile mechanisms used for information systems security enforcement. An access control model formally describes how to decide whether an access request should be granted or denied. Since the rolebased access control initiative has been proposed in the 90s, several access control models have been studied in the literature.

An access control policy is an instance of a model. It defines the set of basic facts used in the decision process. Policies must satisfy a set of constraints defined in the model, which reflect some high level organization requirements. First-order logic has been advocated for some time as a suitable framework for access control models. Many frameworks have been proposed, focusing mainly on expressing complex access control models. However, though formally expressed, constraints are not defined in a unified language that could lead to some well-founded and generic enforcement procedures.

Therefore, we make a clear distinction by proposing a logical framework focusing primarily on constraints, while keeping as much as possible a unified way of expressing constraints, policies, models, and reference monitors. This framework is closely tied to relational database integrity models. We then show how to use well-founded procedures in order to enforce and check constraints. Without requiring any rewriting previous to the inference process, these procedures provide clean and intuitive debugging traces for administrators. This approach is a step toward bridging the gap between general but hard to maintain formalisms and effective but insufficiently general ones.
\end{abstract}

\author{
R. Thion \\ Université de Lyon, \\ Université Lyon 1, LIRIS, UMR5205, F-69622, France \\ Tel. $(+33) 472447936$ \\ Fax. $(+33) 472431536$ \\ E-mail: romuald.thion@univ-lyon1.fr \\ S. Coulondre (Corresponding Author) \\ Université de Lyon, \\ INSA-Lyon, LIRIS, UMR5205, F-69621, France \\ Tel. $(+33) 472437055$ \\ Fax. $(+33) 472438713$ \\ E-mail: stephane.coulondre@insa-lyon.fr
}


Keywords Integrity Model · Dependencies · Authorization · Access Control · Inference

\section{Introduction}

1.1 Access control models, policies and constraints

Security policies are sets of laws and rules governing the security of organizations. They can cover areas from internal organizations rules to national laws, from structural (e.g., fire protection) to organizational aspects (e.g., emergency phone lines). An Access Control (AC) (or authorization) policy is a specialized form of security policy, dedicated to permission management. AC aims at enforcing confidentiality and data integrity.

Within information systems, an AC policy is structured according to an AC model, which formally describes the structure of the policy. A model defines how to decide whether an access request (i.e., an action on an object issued by a subject pertaining to a user) should be granted or denied using a set of rules. For instance, in the Role-Based Access Control (RBAC) models family, roles are assigned to users, and permissions are assigned to roles (Sandhu et al., 1996; Ferraiolo et al., 2003). An RBAC policy is a set of assignments between users and roles and between roles and permissions. The core rule of the RBAC models family states that an access request is granted if and only if the issuer endorses a role with this privilege.

Since the RBAC initiative, several models have been studied in the literature. These models have extended RBAC (e.g., Generalized-Temporal-RBAC (Joshi et al., 2005) or Geographical-RBAC (Damiani et al., 2007)), and have organized policies by additional concepts to enhance their expressive power and flexibility (e.g., WorkflowRBAC (Wainer et al., 2003, 2007), Team-BAC (Thomas, 1997), Task-BAC (Thomas and Sandhu, 1997), Organization-BAC (Miège, 2005)). Throughout these propositions, First-Order Logic (FOL) has been advocated as a general framework suitable to formalize AC models and policies.

In addition to innovative concepts and relations (e.g., roles and hierarchies) for organizing policies, AC models have integrated the concept of constraints. Constraints reflect some high level organization requirements that must be enforced within policies. With the development of $\mathrm{AC}$ models, several kinds of constraints have been defined. The most prominent one is the mutual exclusion, which has been proposed in order to enforce separation of duties ( $\mathrm{Li}$ et al., 2004). Other kinds of constraints have been defined: some mutual exclusion variants, prerequisite constraints or constraints over hierarchies (Crampton, 2003; Jaeger and Tidswell, 2001).

Actually, constraints may express different requirements on policies. Generally speaking, constraints are policy properties that can be classified in the following basic classes:

- (conditional) absence of values (e.g., mutual exclusion),

- (conditional) existence of (constrained) values (e.g., prerequisite),

- (conditional) uniqueness of values (e.g., uniqueness of ancestor in a hierarchy).

These different classes of constraints share a common objective, which is to restrict the set of policies expressible over a model to the set of consistent ones. For instance, in the RBAC setting, the definition of roles $a$ and $b$ as mutually exclusive means that no user should be assigned to $a$ and $b$. Thus, the set of expressible policies is restricted to the set of policies in which no user is assigned to both $a$ and $b$. 
1.2 Problem statement and contribution

Constraints attempt to ensure that policies are consistent. Verifying the consistency of policies is a paramount task. Indeed an inconsistent policy can lead to an unexpected behaviour of the AC system that may completely ruin the benefits of the AC system:

- illegitimate access: exclusion constraints aim at ensuring that some activities are carried out by different users, for instance to prevent that the same user from initiating a payment and authorizing a payment. If a policy does not satisfy the exclusion constraints, one unique user may be able to circumvent the separation of duties and gain unlegitimate access,

- deny of legitimate access: conversely, a constraint can express that every user has the right to $\log$ into the system. Failing to verify such a constraint may lead to users to be unable to do anything,

- difference between expected behaviour and real behaviour: some constraints are implicitly expected from a model. For instance, when dealing with a role hierarchy, it is relevant and quite natural to prevent graph cycles. Failing to verify such constraint may lead to circular inheritance that implies that all the roles have the same set of permissions. Moreover, in a RBAC setting, such a set would be the union of all permissions assigned to at least one role in the cycle,

- unpredictable behaviour: in the worst case, if core constraints like basic integrity requirements of policy (e.g., uniqueness of user related to a subject) are not satisfied, the whole system may be compromised.

In order to express these constraints in an homogeneous way, a formal language able to handle broad classes of constraints is necessary. It should allow the definition of new classes of constraints and should be able to capture general integrity requirements of AC models. This language must have clear semantics, and provide well-founded automated proof procedures for consistency checking.

To address these issues we adopt a top-down approach, starting with a framework focusing primarily on constraints. This framework relies on database integrity theory. We have chosen to present it in a logical setting, but other formalisms, such as tableaux, could have been used. AC models and policies are then formally defined using one of the Datalog languages, which is also a fragment of the database integrity language. This interesting property allows for expressing the whole AC system, including models, policies and constraints, in a single and homogeneous framework. AC models foundations and semantics are described in section 2. Without being universal, the obtained framework can still express several classical models and extensions found in the literature.

We then define AC constraints in section 3. We make use of relational data dependencies, in order to model AC constraints. Dependencies are able to capture complex integrity requirements in an homogeneous way. For instance, one of the properties considered as fundamental in the RBAC standard (Ferraiolo et al., 2003, property 3.2, p.60) (this property is quoted in section 3.5) can be modelled by means of data dependencies. To the best of our knowledge, the property (3.2) has not been modelled and taken into account in any other logical framework for AC.

In section 4, we define a set of well-founded operations that can be used to help AC models designers and policy administrators in making constraints design and administration easier. These operations are generic and can be used over any model built upon the structure defined in sections 2 and 3. 
We have implemented the framework and validated our approach with automated formal proofs based on previous results in the literature that had been manually proved. These results are presented in section 4.6. Moreover, the proofs obtained are quite readable as no prior rewriting is performed (such as clausal form), for it may obfuscate human analysis.

Our approach tries to encompasses many concepts found in the AC literature, but it does not take into account some peculiarities of very dedicated models. For instance, our framework does not encompass authentication (Jim, 2001) and delegation (Li et al., 2003; Wainer et al., 2007). Section 6 evaluates the major design decisions of the framework. The very last section concludes this paper and gives the main directions for future work. For sake of clarity and applicability, most of the examples of in this paper are based upon the RBAC model and further extensions.

\section{Access Control Framework}

This section defines an access control framework able to express several classical models and extensions found in the literature. As we will see in the next section, this framework is a subset of the general framework proposed for access control constraints, that relies on database integrity theory.

Without lack of generality, we operate a clear distinction between models and policies: AC models defines structures and AC policies are instances of these structures. In such a perspective, AC design is the task of defining AC models, whereas administration of $\mathrm{AC}$ is the task of defining policies, which is up to administrators.

FOL has been advocated a suitable formal framework to formalize AC models and policies (Li et al., 2003; Jim, 2001; DeTreville, 2002; Bertino et al., 2003; Li and Mitchell, 2003; Barker and Stuckey, 2003; Halpern and Weissman, 2003; Miège, 2005). From the logical point of view, an AC model both define :

- a vocabulary, i.e., the set of sorts and relations between sorts used to organize access privileges (e.g, subjects, roles, permissions, assignments of roles to subjects)

- a set of so-called rules expressed in a first-order language built over the vocabulary, i.e., the policy that states how privileges are derived from base concepts and relations (e.g., a subject is granted some access only if it endorses some role with the corresponding access right).

Section 2.1 defines the vocabulary and states of an AC model. Section 2.2 defines rules and policies. The generic definition of an $\mathrm{AC}$ model is summarized in section 2.3.

\subsection{Access control vocabulary and state}

An AC model relies on vocabulary: a set of sorts and relations between sorts. Sorts are the main concepts used to organize rights (e.g., users and roles...). Relations define how sorts are related in the model (e.g., assignments between users and roles). The goal of an AC system is to determine whether an access (an action on an object) issued by a subject that represents a user is granted or not. Thus, the main sorts of subjects, users, actions and objects have to be defined in any AC model.

Sorts partition the set of constants in a policy. This property is tied to the manysorted FOL framework. It is shown that many-sorted FOL can be reduced to onesorted Fol (i.e., classical logic) by assigning a specific unary predicate symbol $D_{S}$ 


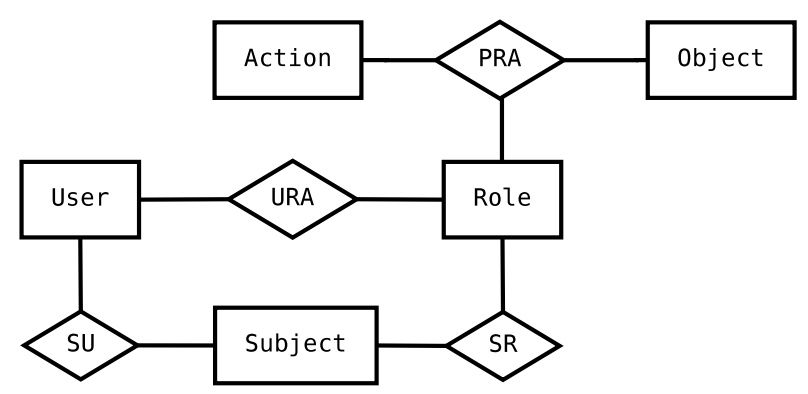

Fig. 1 The core vocabulary edb of RBAC.

called domain predicate symbols to each sort $S$ (Gallier, 1986, chapter 10, p. 460). In our framework, we implicitly operate this transformation by assigning a unique unary predicate symbol to each sort. The vocabulary is represented in the relational formalism according to the standard terminology used in databases.

Definition 1 Access control vocabulary. The vocabulary Voc of an $\mathrm{AC}$ model is the union of a set Sorts of unary predicate symbols called sorts and a set Rels of $n$-ary predicate symbols called relations.

The sorts of users (User), subjects (Subject), actions (Action) and objects (Object) must be present in any $\mathrm{AC}$ vocabulary.

Figure 1 illustrates the core vocabulary of RBAC models. This vocabulary is composed of five sorts (drawn by rectangles in figure 1): User, Subject, Role, Action and Object. Moreover, four relations are defined (drawn by diamonds in figure 1): URA betwen $U$ ser and Role, PRA between Role, Action and Object, SU between Subject and $U$ ser and $S R$ between Subject and Role.

We define the state of an AC model, which is a set of facts defined over the core vocabulary $e d b$. The state of an AC model is the extensive part of a policy, which can practically be stored in a Relational Database Management System (RDBMS). Following traditional axioms of logical interpretation of relational databases, we assume that constants are distinct and that states are finite.

Definition 2 Access control state. To each sort $S \in$ Sorts is associated a set of constants $\mathbf{S}$ called its domain. Domains are pairwise disjoint: $\mathbf{S} \cap \mathbf{S}^{\prime}=\emptyset$ for all $S \neq S^{\prime}$ in Sorts. To each relation $R \in$ Rels of arity $n$ between sorts $S_{1} \ldots S_{n}$, is associated the set $\mathbf{R}=\mathbf{S}_{1} \times \ldots \times \mathbf{S}_{n}$.

An access control state $\mathbf{I}$ on an AC vocabulary Voc is a mapping from each sort $S \in$ Sorts to a finite subset of $\mathbf{S}$, called its active domain, and from each relation $R \in$ Rels to a finite subset of $\mathbf{R}$.

In the context of RBAC, the term AC state (a.k.a. RBAC database) has first been coined by (Gavrila and Barkley, 1998). A toy sample of an RBAC state I (over vocabulary shown in figure 1) is given in table 1 . In this state, the sort $U$ ser takes its values from the set $\{$ Alice, Bob, Charly $\}$, Role from $\left\{\mathrm{r}_{1}, \mathrm{r}_{2}, \mathrm{r}_{3}, \mathrm{r}_{4}\right\}$, Action from $\{\mathrm{r}, \mathrm{w}, \mathrm{x}\}$ and Object from $\{$ file1, file2, file $\}$

In this state, Bob is assigned both roles $r_{1}$ and $r_{3}$ and Bob endorses these roles in two different sessions, namely $\mathrm{S} 2$ and $\mathrm{S} 3$. The rules of RBAC models allow inferring that Bob can read file1, file 2 and file 3 as the role $r_{1}$ is granted $r$ access on file1, file2 and file3. 


\begin{tabular}{lr}
\multicolumn{2}{c}{$U R A$} \\
\hline User & Role \\
\hline Alice & $\mathrm{r}_{1}$ \\
Alice & $\mathrm{r}_{2}$ \\
Bob & $\mathrm{r}_{1}$ \\
Bob & $\mathrm{r}_{3}$ \\
Charly & $\mathrm{r}_{1}$ \\
Charly & $\mathrm{r}_{4}$ \\
\hline & \\
& \\
\hline Subject & $\mathrm{Role}$ \\
\hline S1 & $\mathrm{r}_{1}$ \\
S1 & $\mathrm{r}_{2}$ \\
S2 & $\mathrm{r}_{1}$ \\
S3 & $\mathrm{r}_{3}$ \\
S4 & $\mathrm{r}_{4}$ \\
\hline
\end{tabular}

\begin{tabular}{lcr}
\multicolumn{3}{c}{$P R A$} \\
\hline Role & Action & Object \\
\hline$r_{1}$ & $r$ & file1 \\
$r_{1}$ & $r$ & file2 \\
$r_{1}$ & $r$ & file3 \\
$r_{2}$ & w & file1 \\
$r_{3}$ & w & file2 \\
$r_{3}$ & r & file4 \\
$r_{3}$ & w & file4 \\
$r_{3}$ & $x$ & file4 \\
$r_{4}$ & w & file3 \\
$r_{4}$ & $r$ & file4 \\
$r_{4}$ & w & file4 \\
$r_{4}$ & $x$ & file4 \\
\hline & $S U$ & User \\
\hline Subject & & Alice \\
\hline S1 & & Bob \\
S2 & & Bob \\
S3 & & Charly \\
S4 & &
\end{tabular}

Table 1 A sample RBac state.

2.2 Access control rules and policies

In this section we define the $\mathrm{AC}$ rules. Rules express the deductive principles of an $\mathrm{AC}$ model. From a set of rules $P$ and a state $\mathbf{I}$, it is possible to compute derived relations on which the access decision process is based. The state is the minimal knowledge from which a complete policy may be derived using the set of rules.

For instance, in order to prevent administrators from inserting redundancies in a policy, several algebraic properties of relations are commonly assumed: transitivity, reflexivity or symmetry for instance. These properties are expressed in intenso by means of rules. Thus, administators only have to insert the minimal knowledge in the state. The complete policy is obtained by applying the set of rules $P$ as a closure operator producing new facts.

In the case of complex AC models, defining derived relations within the model is a mandatory prerequisite in order to obtain a maintainable state. This separation between extensive relations in the state and intensive relations in the policy is a design choice made according to the recommendations addressed to the RBAC standard (Li et al., 2007), in particular the third suggestion:

Suggestion 3: "The standard should make a clear distinction between base relations and derived relations."

Several fragments of FOL have been used as formal languages for modelling AC rules. DATALOG-based models are considered expressive enough to capture complex AC policies (Bertino et al., 2003), (Miège, 2005), (Jim, 2001), (Barker and Stuckey, 2003). We focused our work on FOL rules of DATALOG ${ }^{C}$, which has been recognized as fruitful to formalize AC models ( $\mathrm{Li}$ and Mitchell, 2003). 
We operate the main following specializations on Fol by selecting DATALOG ${ }^{C}$ as a formal language for rules: functions, negation and disjunction are not allowed. Formally, DATALOG ${ }^{C}$ sentences are formal expressions of the form:

$$
\forall \tilde{X} R_{1}\left(X_{1}\right) \wedge \ldots \wedge R_{n}\left(X_{n}\right) \wedge \psi(\tilde{X}) \Rightarrow R_{0}\left(X_{0}\right)
$$

where $\forall i \in 0 . . n, R_{i}$ are symbols from $V o c$ and $X_{i}$ are sequences of logic variables of length equal to the arity of $R_{i}$. The left-hand side of a rule $\left(R_{1}\left(X_{1}\right) \wedge \ldots \wedge R_{n}\left(X_{n}\right) \wedge\right.$ $\psi(\tilde{X}))$ is called the body of the rule its right-hand side $\left(R_{0}\left(X_{0}\right)\right)$ is called its head. $\tilde{X}$ is the set of variables that appear in the body. $\psi(\tilde{X})$ is a conjunction of linear arithmetic constraints (e.g., $\leq, \geq,=, \neq$ ) over variables and constants of same sorts.

Definition 3 Access control rules. The rules of and AC model is a set $P$ of DATALOG $C$ sentences.

$P$ defines a partition on the vocabulary $V o c: i d b \cap e d b=\emptyset$ and $i d b \cup e d b=V o c$. $i d b$, for intensive database, is the set of relations that appear in the heads of the rules and $e d b$, for extensive database, is the set of relations that appear only in the bodies of the rules but not in their heads.

The definition of the component $P$ that captures rules is generic. It encompasses the main notions introduced in the literature to organize AC policies. Next subsections focus on three main derived relations that are commonly used in AC models : authorization triple, hierarchies and mutual exclusion. We can now formally define AC policies.

Definition 4 Access control policy. The semantics of rules is given by the standard FOL model-theoretic interpretation of DATALOG ${ }^{C}$ rules.

Given an $\mathrm{AC}$ state $\mathbf{I}$ of a model $A C=(V o c, P)$, an access control policy $\mathbf{I}^{\prime}$ over a given state $\mathbf{I}$ is a logical model of $P$ stated $\mathbf{I}^{\prime} \models P$ with $\mathbf{I} \subseteq \mathbf{I}^{\prime}$.

The existence of a decidable procedure which computes the interpretation $\mathbf{I}^{\prime}$ from I ensures that it is always possible to compute the policy and consequently to answer whether an access request is granted or denied. DATALOG's (and its major extension such as DATALOG ${ }^{C}, \mathrm{C}$-DATALOG or DATALOG $\urcorner$ ) restrictions ensure that there is a unique minimal model of $P$ and that this model can be computed in a finite time (Abiteboul et al., 1995, theorem 12.5.2, p. 301). Uniqueness of the minimal model ensures that for given state and set of rules there is a unique derived policy. The finite time property ensures that computation of the policy if decidable and thus, it ensures that access control requests will be always answered.

\subsubsection{Derivation of authorizations}

We have defined the structure of an AC model, however deriving authorizations from state has not been explained yet. As coined by Lampson in his seminal paper (Lampson, 1974), the aim of access control is to take a boolean AC decision upon an AC request. An $\mathrm{AC}$ request is a triple subject, action, and object. The reference monitor which enforces $\mathrm{AC}$ acts as a Non-bypassable, Evaluatable, Always Invoked and Tamperproof $\left(\mathrm{NEAT}^{1}\right)$ proxy between subjects and objects. It takes authorization decisions upon the policy.

\footnotetext{
1 http://www.ois.com/Products/MILS-Technical-Primer.html
} 


\begin{tabular}{rll}
\hline$S R(S, R) \wedge P R A(R, A, O)$ & $\Rightarrow$ & $\operatorname{Access}(S, A, O)$ \\
$U R A(U, R) \wedge P R A(R, A, O)$ & $\Rightarrow$ & $\operatorname{Static}(U, A, O)$ \\
$S U(S, U) \wedge \operatorname{Access}(S, A, O)$ & $\Rightarrow$ & $\operatorname{Dynamic}(U, A, O)$ \\
\hline
\end{tabular}

Table 2 Rules for fundamental triples in RBAC.

Definition 5 Fundamental triples. The three following fundamental triples symbols must appear in the intensive database $i d b$, and the set of rules $P$ must define how to derive the corresponding relations Access $\subseteq$ Subject $\times$ Action $\times$ Object, Static $\subseteq$ User $\times$ Action $\times$ Object and Dynamic $\subseteq$ User $\times$ Action $\times$ Object.

The relation Access is the set of $\mathrm{AC}$ permissions granted to subjects. Static is the set of permissions granted to users independently of the subjects they use. Dynamic is the set of permissions granted to users through the subjects. The inclusion Dynamic $\subseteq$ Static must hold in any model.

Authorizations decisions are based upon triples Access derived from the state. Thus, the reference monitor can be modelled as a function:

$$
\begin{aligned}
& \mathcal{F}: \text { Subject } \times \text { Action } \times \text { Object } \rightarrow\{\text { false }, \text { true }\} \\
& \mathcal{F}(s, a, o)=\left\{\begin{array}{l}
\text { true if }(s, a, o) \in \text { Access } \\
\text { false otherwise }
\end{array}\right.
\end{aligned}
$$

The three rules showed in table 2 define the triples in the RBAC model. According to the sample toy state of table 1 , user Bob is assigned to two roles $r_{1}$ and $r_{3}$. One may be interested by the authorizations statically granted to Bob which is $\left\{\left(r, f i l e_{1}\right),\left(r, f i l e_{2}\right),\left(r, f i l e_{3}\right),\left(w, f i l e_{2}\right),\left(r, f i l e_{4}\right),\left(w, f i l e_{4}\right),\left(x, f i l e_{4}\right)\right\}$ by means of the query:

$$
\{(a, o) \mid \operatorname{Static}(\text { Bob }, a, o)\}
$$

\subsubsection{AC models hierarchies}

The RBAC standard contains two major features to make AC administration easier: role hierarchies and constraints. Hierarchies are a way to reduce redundant user and permission-role assignments. Roles are given a preorder (reflexive, transitive) $\preccurlyeq$ modelling an is a relationship. Relation $r_{1} \preccurlyeq r_{2}$ means that every permissions granted to role $r_{1}$ are granted to $r_{2}$ and that each user who is a member of role $r_{2}$ is also a member of $r_{1}$. Our framework generalizes this approach and takes recommendations of $\mathrm{Li}$ et al. (2007) into account:

Suggestion 4: "The Reference Model should maintain a relation that contains the role dominance relationships that have been explicitly added, and update this relation when the role hierarchy changes."

For any sort $S \in e d b$ of an AC model, we define two relations: a dominance relation Senior $D_{S} \in e d b$ stored in the state, and an inheritance relation Senior $_{S} \in i d b$ defined as the reflexive transitive closure of $S e n i o r D_{S}$. Please note that we use suffix $D$ to distinguish between extensive and intensive predicate.

Definition 6 Inheritance. A sort $S \in e d b$ of an AC model is given an inheritance relationship if a dominance relation Senior $D_{S}$ with Senior $D_{S} \subseteq S \times S$ is defined in edb and if the following three rules are defined in $P$ : 


$$
\begin{aligned}
& \text { Senior } D_{S}\left(S, S^{\prime}\right) \quad \Rightarrow \quad \operatorname{Senior}_{S}\left(S, S^{\prime}\right)
\end{aligned}
$$

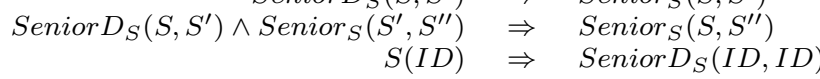

If defined, a Senior $_{S}$ relation should be used in the definition of AC triples. For instance, in the $\mathrm{RBAC}$ models with role hierarchies, the rule deriving the Access relation is redefined as follows, in order to take the role hierarchy into account:

$$
S R(S, R) \wedge \operatorname{Senior}_{R}\left(R, R^{\prime}\right) \wedge P R A\left(R^{\prime}, A, O\right) \Rightarrow \operatorname{Access}(S, A, O)
$$

State from table 1 is an instance of the flat RBAC model, without role hierarchy. Assume that we add $r_{5}$ into roles and that $r_{5}$ inherits $r_{4}$ Senior $R$ Role $\left(r_{5}, r_{1}\right)$. With the extended Access rule, any user who is assigned to $r_{5}$ has at least $r$ access on file1, file2 and file3 because these permissions are granted to $r_{1}$.

\subsubsection{Mutual exclusion relation}

Another important feature introduced in the AC literature is mutual exclusion, which is the main constraint defined in the RBAC standard (Ferraiolo et al., 2003). For instance, one may define that no user can be assigned to both roles $r_{3}$ and $r_{4}$, because they stands for mutually incompatible roles.

As it is the case for hierarchies, mutual exclusion needs two relations: an extensive one $S o D D$ and an intensive one $S o D$ which is its symmetric closure. Moreover, when both an exclusion and an inheritance relation have been defined on the same sort, an additional rule must be defined in $P$, to ensure that exclusion is propagated via inheritance (Gavrila and Barkley, 1998).

Definition 7 Mutual exclusion. A sort $S \in e d b$ is given a mutual exclusion relationship if a core separation relation $S o D D_{C} \subseteq S \times S$ is defined in edb and if a relation $S_{S} D_{S} \subseteq S \times S$ is defined with the following two principles in the set of rules $P$ :

$$
\begin{array}{rll}
\operatorname{SoDD}_{S}\left(S, S^{\prime}\right) & \Rightarrow & \operatorname{SoD}_{S}\left(S, S^{\prime}\right) \\
\operatorname{SoD}_{S}\left(S, S^{\prime}\right) & \Rightarrow & \operatorname{SoD}_{S}\left(S^{\prime}, S\right)
\end{array}
$$

If an inheritance relation Senior $_{S}$ is also set on a sort $S$, then the following rule must be defined:

$$
\operatorname{SoD}_{S}\left(S, S^{\prime}\right) \wedge \operatorname{Senior}_{S}\left(S^{\prime \prime}, S\right) \Rightarrow \operatorname{SoD}_{S}\left(S, S^{\prime \prime}\right)
$$

Last definition is still incomplete: we have not defined which constraint the mutual exclusion relation really enforces. We have neither expressed that an inheritance relation should be antisymmetric nor that a subject should be assigned to a unique user. As a matter of fact, we have only expressed how to define the policy $\mathbf{I}^{\prime}$. We will show in the next section how to define the conditions upon which the policies are consistent according to a set of constraints.

For instance, in the toy RBAC state of table 1, one states that $r_{3}$ and $r_{4}$ are mutually exclusive by adding $S o D_{\text {Role }}\left(r_{3}, r_{4}\right)$ into the state. It may be a way to ensure that request (allowed to $r_{3}$ ) and approval (allowed to $r_{4}$ ) of major expenditure are done by two separate people. In the toy RBAC state, it should be inconsitent to state $S o D_{\text {Role }}\left(\mathrm{r}_{1}, \mathrm{r}_{3}\right)$ because user Bob is assigned to both. 
2.3 Framework summary

Policies are logical models (in the model-theoretic sense) of a theory defined by an AC model. The word model is indeed prone to confusion. We use the term (AC) model to refer to the structure that describes how rights are organized and granted (i.e., the meaning of model in the AC literature). We explicitly use the term logical model to refer to a model-theoretic interpretation which satisfies a set of closed FOL formulae.

This preliminary modelling step is paramount for addressing further issues. The proposed framework is closely related to the deductive database paradigm. In this paradgim, a database is defined with a schema (i.e., the AC vocabulary $V o c$ ) and a set of deduction rules (i.e., expressed in DATALOG).

An AC state is considered as a set of relational data structured accordingly to a given vocabulary. A policy is a set of facts derived from a state and a set of rules. Formally, we have defined an $\mathrm{AC}$ model as a pair $A C=(V o c, P)$ composed as follows:

- Voc, the access control vocabulary: a set of unary relations called sorts and $n$-ary relations between these sorts. This vocabulary sets the core of the AC model used to organize the policies. An intepretation $\mathbf{I}$ of edb is a state.

- P: a set of Datalog ${ }^{C}$ rules defining the AC model rules. These rules allow deducing consequent facts from $\mathbf{I}$, thus defining an intensive vocabulary $i d b$ and a policy $\mathbf{I}^{\prime}$ over $i d b$.

One of our main objectives is to treat constraints as first class citizens. In the next section, we will extend the definition of (simple) AC model to be a triple $A C=$ $(V o c, P, \Sigma)$. This refinement includes a set $\Sigma$ of Fol formulae modelling the AC model constraints. They are expressed by data dependencies, which subsume the expressive power of traditional deductive database such as DATALOG ${ }^{C}$. As shown in this paper, this enhanced expressivity is needed to model complex integrity constraints found in AC models. Whereas formulae of $P$ allow deducing the policy from a given state, those from $\Sigma$ restrict $\mathbf{I}$ and $\mathbf{I}^{\prime}$. The definition and usage of $\Sigma$ are defined in the next section.

\section{Access Control Constraints}

Among the formal tools available in the database area, data dependencies (a.k.a integrity constraints) have been defined to capture integrity requirements on relational data. In a unification attempt, they have been defined as Fol sentences (Abiteboul et al., 1995, Chapter 10). In the proposed framework, dependencies are used to capture AC constraints: they capture formal integrity requirements of policies.

\subsection{Data dependencies}

Dependencies share common characteristics with DATALOG deduction rules, but they form a larger subclass of FOL sentences. Data dependencies are categorized into classes of increasing expressivity. The best known classes are functional (FD), inclusion (IND) and multivalued (MVD) dependencies (Abiteboul et al., 1995). Expressive classes have been developed to express complex statements on relational data. They can model semantic relationships in spatial, temporal or multimedia databases. 
One of the most general form of dependencies is constrained tuple-generating dependencies (CTGD) (Maher and Srivastava, 1996) which are Fol sentences having the following syntax (we reuse convention from section 2.2):

$$
\begin{aligned}
& \forall \tilde{X} R_{1}\left(X_{1}\right) \wedge \ldots \wedge R_{n}\left(X_{n}\right) \wedge \psi(\tilde{X}) \Rightarrow \\
& \exists \tilde{Z} Q_{1}\left(Y_{1}\right) \wedge \ldots \wedge Q_{m}\left(Y_{m}\right) \wedge \phi(\tilde{Y})
\end{aligned}
$$

where $\tilde{Z}$ does not designate the whole set of variables in the head but only those which are not already bound by a universal quantifier $(\tilde{Z}=\tilde{Y}-\tilde{X})$.

Special forms of dependencies considered in this paper are restriction of CTGD:

1. Constraint-Generating Dependencies (CGD), head is restricted to constraints:

$$
\forall \tilde{X} R_{1}\left(X_{1}\right) \wedge \ldots \wedge R_{n}\left(X_{n}\right) \wedge \psi(\tilde{X}) \Rightarrow \phi(\tilde{X})
$$

2. Nullity-Generating Dependencies (NGD), head is empty ${ }^{2}$ :

$$
\forall \tilde{X} R_{1}\left(X_{1}\right) \wedge \ldots \wedge R_{n}\left(X_{n}\right) \wedge \phi(\tilde{X}) \Rightarrow \perp
$$

3. Full Tuple-Generating Dependencies or Total Tuple-Generating Dependencies (FullTGD) do not include existentially quantified variables:

$$
\forall \tilde{X} R_{1}\left(X_{1}\right) \wedge \ldots \wedge R_{n}\left(X_{n}\right) \Rightarrow Q_{1}\left(X_{1}^{\prime}\right) \wedge \ldots \wedge Q_{m}\left(X_{m}^{\prime}\right)
$$

4. Tuple-Generating Dependencies (TGD) or Generalized Dependencies, generalize both FTGD and IND but do not allow constraints:

$$
\forall \tilde{X} R_{1}\left(X_{1}\right) \wedge \ldots \wedge R_{n}\left(X_{n}\right) \Rightarrow \exists \tilde{Z} Q_{1}\left(Y_{1}\right) \wedge \ldots \wedge Q_{m}\left(Y_{m}\right)
$$

Dependencies are used either to restrict authorized values in a policy when their heads do not include atoms (e.g., CGD, NGD), or to impose presence of tuples if some other ones are already present in the policy (e.g., IND, MVD, TGD). Note that NGD allow the expression of negative requirements as $\phi \Rightarrow \perp$ is logically equivalent to $\neg \phi$.

In our framework, the set of constraints over an $\mathrm{AC}$ model is denoted as $\Sigma$. It captures conditions that must hold in the policy. The expressivity of dependencies is needed to capture desirable properties which cannot be expressed in $P$, which relies on the DAtALOG ${ }^{C}$ fragment, mainly due to the absence of existential quantifier. From the logical perspective the $P \cup \Sigma$ of rules and constraints is a logical theory, i.e., a set of closed FoL formulae.

Definition 8 Access control model. An access control model is a triple $A C=(V o c, P, \Sigma)$, where $\Sigma$ is a set of constraints expressed as data dependencies.

The semantics of constraints is given by the standard FOL model-theoretic interpretation of dependencies. An access control policy $\mathbf{I}^{\prime}$ built from a state $\mathbf{I}$ is consistent iff $\mathbf{I}^{\prime}=\Sigma$.

The next subsections describe how dependencies are used to express various security requirements of models in an homogeneous way: integrity of states, algebraic properties, semantics of mutual exclusion, constraints on authorizations and administrative prerequisites.

\footnotetext{
$2 \perp$ stands for a logical antilogy (e.g., $0=1)$
} 


\begin{tabular}{rll}
\hline$S R(S, R)$ & $\Rightarrow$ & $\exists U S U(S, U)$ \\
$S U(S, U) \wedge S U\left(S, U^{\prime}\right)$ & $\Rightarrow$ & $U=U^{\prime}$ \\
$S U(S, U) \wedge S R(S, R)$ & $\Rightarrow$ & $U R A(U, R)$ \\
\hline
\end{tabular}

Table 3 Integrity requirements for $S U$ relation in RBAC.

3.2 Integrity constraints on AC states

We define a category of constraints ensuring that relations are well-founded. For example, whenever a relation over sorts exists, the sorts must exist too. Thus, the sentence $U R A(U, R) \Rightarrow U \operatorname{ser}(U) \wedge \operatorname{Role}(R)$ should be enforced in any RBAC policies: a role can be assigned to a user only if both the user and the role exist. This can be considered as an equivalent to foreign key constraints in RDBMS. These kinds of constraints ensure that what is actually stored is consistent. This leads to the definition of well-founded state.

Definition 9 Well-founded state. Let $\Lambda$ be a set of constraints $\Lambda \subseteq \Sigma$ involving only symbols of the extensive database edb, such that for each $n$-ary relation $R \in$ edb over sorts $S_{1} \ldots S_{n} \in e d b, \Lambda$ contains an inclusion dependency of the form:

$$
R\left(I D_{1}, \ldots, I D_{n}\right) \Rightarrow S_{1}\left(I D_{1}\right) \wedge \ldots \wedge S_{n}\left(I D_{n}\right)
$$

Let $\mathbf{I}$ be a state. Then $\mathbf{I}$ is well-founded if $\mathbf{I} \models \Lambda$.

For instance, in the RBAC standard it is defined that each subject has to be assigned to a unique user and that a role can be used by a subject only if the role is assigned to the user who owns the subject. These constraints can be defined by dependencies as shown in table 3 . These requirements are satisfied by the RBAC state given in table 1 , thus this state is a well-founded one.

The dependencies paradigm allows the formalization of the next suggestion found in the critique of the RBAC standard (Li et al., 2007):

Suggestion 2: "The standard should accommodate RBAC systems that allow only one role to be activated in a session"

This constraints can be captured in a straightforward way by means of a functional dependency that enforces a key constraint $S R(U, R) \wedge S R\left(U, R^{\prime}\right) \Rightarrow R=R^{\prime}$. Actually, this requirement is not satisfied by table 1 , because Alice endorses two different roles in session $\mathrm{S} 1$.

\subsection{Algebraic constraints of relations}

AC models hierarchies are commonly defined as partial orders for avoiding cycles (e.g., (Kuhn, 1997) for RBAC or (Miège, 2005) for OrBAC). Moreover, a mutual exclusion is defined as irreflexive to prevent a sort from being mutually exclusive with itself (e.g., (Li et al., 2004)). As example, antisymmetry of inheritance relation and irreflexivity of mutual exclusion in RBAC can be expressed by dependencies, as given in table 4 .

Moreover, some additional properties may be desirable. For example some models (e.g., Lattice-BAC (Sandhu, 1993)) organize sorts using forests of trees, forests of inverted trees or lattices (posets in which any two elements have a join and a meet). 
These additional requirements over Senior $_{S}$ and Senior $_{S}$ relations can be expressed by CGD. Note that the restriction of a hierarchy to a lattice could not be expressed in DATALOG-based framework, because these frameworks lacks existentially quantified variables, and the property states that there exists some meet and join for any given pair of elements.

Definition 10 Restricted hierarchies. Let Senior ${ }_{S} \in i d b$ be an intensive inheritance relation computed from an extensive one Senior $D_{S} \in e d b$. Senior ${ }_{S}$ is called a limited hierarchy of type:

- forest of trees, if $\Sigma$ contains the following FD:

$$
\text { Senior } D_{S}\left(S, S^{\prime}\right), \text { Senior } D_{S}\left(S, S^{\prime \prime}\right) \Rightarrow S^{\prime}=S^{\prime \prime}
$$

- forest of inverted trees, if $\Sigma$ contains the following FD:

$$
\text { Senior } D_{S}\left(S^{\prime}, S\right), \text { Senior } D_{S}\left(S^{\prime \prime}, S\right) \Rightarrow S^{\prime}=S^{\prime \prime}
$$

- lattice, if $\Sigma$ contains the following FTGD:

$$
\begin{aligned}
& S\left(S^{\prime}\right), S\left(S^{\prime \prime}\right) \Rightarrow \exists S_{\perp} \operatorname{Senior}_{S}\left(S^{\prime}, S_{\perp}\right), \text { Senior }_{S}\left(S^{\prime \prime}, S_{\perp}\right) \\
& S\left(S^{\prime}\right), S\left(S^{\prime \prime}\right) \Rightarrow \exists S_{\top} \operatorname{Senior}_{S}\left(S_{\top}, S^{\prime}\right), \text { Senior }_{S}\left(S_{\top}, S^{\prime \prime}\right)
\end{aligned}
$$

\subsection{Semantics of mutual exclusion}

Although the intensive $S o D_{S} \in i d b$ relation ensures that there is a mutual exclusion on sort $S$, the principles of mutual exclusion can be applied from different perspectives. In the RBAC model, if two roles $r$ and $r^{\prime}$ are stated as mutually exclusive, several interpretations can exist (Crampton, 2003). All these different semantics can be stated respectively by means of CGD:

- no user could be assigned to both roles $r$ and $r^{\prime}$ :

$$
\operatorname{SoD}_{\text {Role }}\left(R, R^{\prime}\right) \wedge U R A(U, R) \wedge U R A\left(U, R^{\prime}\right) \Rightarrow \perp
$$

- no subject could be assigned to both $r$ and $r^{\prime}$ :

$$
S o D_{\text {Role }}\left(R, R^{\prime}\right) \wedge S R(S, R) \wedge S R\left(S, R^{\prime}\right) \Rightarrow \perp
$$

- no common permission could be granted to both $r$ and $r^{\prime}$ :

$$
\operatorname{SoD}_{\text {Role }}(R, R) \wedge P R A(R, A, O) \wedge P R A\left(R^{\prime}, A, O\right) \Rightarrow \perp
$$

- no action over a common object could be granted to both $r$ and $r^{\prime}$ :

$$
\operatorname{SoD}_{\text {Role }}\left(R, R^{\prime}\right) \wedge P R A(R, A, O) \wedge P R A\left(R^{\prime}, A^{\prime}, O\right) \Rightarrow \perp
$$

For instance, according to the first semantics, it is inconsistent to add $S o D_{\text {Role }}\left(\mathrm{r}_{1}, \mathrm{r}_{2}\right)$ in the state given in table 4, because Alice is granted both roles $r_{1}$ and $r_{2}$. According to the second semantics, there is an inconsistency because of session S1. However, according to the third semantics, there is no inconsistency because no permission is granted to two different roles.

$$
\begin{array}{rll}
\operatorname{Senior}_{\text {Role }}\left(R, R^{\prime}\right) \wedge \operatorname{Senior}_{\text {Role }}\left(R^{\prime}, R\right) & \Rightarrow & R=R^{\prime} \\
\operatorname{SoD}_{\text {Role }}(R, R) & \Rightarrow & \perp
\end{array}
$$

Table 4 Antisymmetry of role hierarchy, and irreflexivity of mutual exclusion. 


\begin{tabular}{rll}
\hline $\operatorname{Access}(S, A, O)$ & $\Rightarrow$ & $\exists R S R(S, R) \wedge P R A(R, A, O)$ \\
$\operatorname{Static}(U, A, O)$ & $\Rightarrow$ & $\exists R U R A(U, R) \wedge P R A(R, A, O)$ \\
$\operatorname{Dynamic}(U, A, O)$ & $\Rightarrow$ & $\exists S S U(S, U) \wedge \operatorname{Access}(S, A, O)$
\end{tabular}

Table 5 Constraints on authorizations in RBAC.

3.5 Constraints on authorization relations

The RBAC model imposes that any authorization must be granted via a role (Ferraiolo et al., 2003):

Property 3.2: "A subject $s$ can perform an operation op on object o only if there exists a role $r$ that is included in the subject's active role set and there exists an permission that is assigned to $r$ such that the permission authorizes the performance of op on o".

This property can be considered as fundamental in structured AC models. Defining intermediate sorts between users and permissions is a way to simplify administration tasks. Bypassing these sorts is error-prone and may lead to ambiguities within policies. Thus, we argue that such a property defined for RBAC should be generalized. To the best of our knowledge, this property is neither captured in logic-based modelling attempts of RBAC, nor in extended models.

Definition 11 Property of authorizations. If there is a rule in $P$ with head Access (resp. Static, Dynamic) and hypothesis $\psi$, then there is a corresponding constraint in $\Sigma$ of the form Access $\Rightarrow \psi$ (resp. Static, Dynamic) that makes the rule an if and only if condition.

According to the rules for triples given in table 2, the constraints of table 5 can be derived. These TGD use existentially quantified variables shared among multiple predicates. Such sentences can not be expressed in DATALOG-based frameworks because they lack the existential quantifier. This quantifier is needed to capture so called invented values in the database terminology, i.e., unknown values that have to be present in a policy to ensure its consistency.

\subsection{Administrative prerequisite}

An administrative prerequisite enforces the presence of tuples before allowing administrative operations (Ferraiolo et al., 2003). Administrative operations consist in updates, insertions and deletions of tuples within the state I. If any administrative prerequisite, expressed by some dependencies, is violated, the transaction initiated by the administrator will not be committed.

Definition 12 Administrative prerequisite. An administrative prerequisite constraint imposes the presence of tuples in $\mathbf{I}^{\prime}$. A transitive prerequisite relation Required R $_{R} \in i d b$ over a relation $R$ is defined as the transitive closure of a relation Required $D_{R} \in e d b$. Prerequisite constraints can be modelled as (constrained) TGD in $\Sigma$ by an auxiliary relation Required $D_{R}$ of the following form, where the predicate $R$ is present in both the sentence body and head:

$$
\forall \tilde{X} R\left(X_{R}\right) \wedge \ldots \wedge \text { Required }_{R}(X) \wedge \phi(\tilde{X}) \Rightarrow \exists \tilde{Z} R\left(X_{R}^{\prime}\right) \wedge \ldots \wedge \psi(\tilde{Y})
$$


For instance, in the RBAC models, administrative prerequisites are used for preventing administrators from assigning roles to users if some other role has not already been defined. This is an example of a prerequisite over the relation $U R A$. The following sentences express these statements. Defining Required $\left(r_{1}, r_{2}\right)$ in $\mathbf{I}$ imposes that whenever a user is assigned to $r_{2}$, then there must be at least one user assigned to $r_{2}$.

$$
\begin{aligned}
\text { Required } D\left(R, R^{\prime}\right), \text { Required }\left(R^{\prime}, R^{\prime \prime}\right) & \Rightarrow \quad \text { Required }\left(R, R^{\prime \prime}\right) \\
U R A(U, R), \text { Required }\left(R, R^{\prime}\right) & \Rightarrow \quad \exists U^{\prime} U R A\left(U^{\prime}, R^{\prime}\right)
\end{aligned}
$$

\section{Constraint verification}

From now on, we have defined the three basic components of an AC model. The task of defining the vocabulary $V o c$ (the names of subjects, roles, assignments, etc.), the set of administrative rules $P$ and the set of integrity constraints $\Sigma$ is dedicated to the AC model designer. Administrators define only the state $\mathbf{I}$, as $\mathbf{I}^{\prime}=P(\mathbf{I})$ is computed from $\mathbf{I}$ and $P$. The policy is consistent if $\mathbf{I}^{\prime} \models \Sigma$. Thus, from a logical perspective, there are only a few differences between $P$ and $\Sigma$ which can be considered as a whole as a logical theory $T=P \cup \Sigma$.

\subsection{Formal characterization}

The main difference between $P$ and $\Sigma$ lays in their usage: rules are used for computing the policy from the state, whereas constraints are used to impose restrictions on authorized instances of $\mathbf{I}^{\prime}$. Notice that the uniqueness and computability of $\mathbf{I}^{\prime}$ is ensured by the properties of the fragment of FOL used for $P$.

In this section we rely on two theoretical problems over logical theories:

- the satisfaction problem. Answering whether a policy $\mathbf{I}^{\prime}$ satisfies a given Fol sentence $\sigma: \mathbf{I}^{\prime} \models \sigma$. This problem is central for computing $\mathbf{I}^{\prime}$ from $\mathbf{I}$, for answering queries and for checking whether a policy satisfies the set of constraints for a given model. The satisfaction problem is decidable for the class of formulae used for $A C=(V o c, P, \Sigma)$.

- the logical implication problem. Answering whether a set of closed formulae $T$ logically implies a closed formula $\sigma: T \models \sigma$ or, in other words, deciding if any policy model of a theory $T$ is also a model of the single sentence $\sigma$. This problem is central for simplifying logical theory or for checking model consistency from an abstract perspective, without considering any state. This problem is decidable in the class of FTGD extended with constraints. However, it is semi-decidable (it may not halt for negative answers but will always halt for positive ones) for larger classes of dependencies such as CTGD.

As dependencies such as CTGD are strictly more expressive than DATALOG ${ }^{C}$, and as they share the same Fol semantics, we will actually treat the sentences of $P$ as dependencies to build and homogeneous logical theory $T$ made of rules and constraints. Thus, it is possible to use the same proof procedures for both rules and constraints without distinction.

We have implemented the proof procedures for dependencies presented in (Beeri and Vardi, 1984), (Maher and Srivastava, 1996) and (Coulondre, 2003) to validate 
our approach. As shown in section 4.6, our prototype allows automatizing the consistency checking of RBAC policies and furnishing proof of previous results independently proposed (Gavrila and Barkley, 1998).

\subsection{Administrative review}

One of the features expected from an access control model implementation is the $a d$ ministrative review. This set of operations has been defined in the RBAC standard: "When URA and PRA relation have been created, it should be possible to view the contents of those relations from both the user and role perspectives[...]". In the present framework, administrative reviews are simple conjunctive queries over $\mathbf{I}^{\prime}$. Therefore, the requirements of the standard definition are met.

Definition 13 Administrative reviews. Any conjunctive query (from the standard database sense) built upon the vocabulary Voc is an administrative review.

Examples of queries built upon the RBAC model vocabulary $V o c$ are respectively (1) the set of permissions granted directly (without hierarchy) to users through their roles , (2) the set of users who can execute an object, whatever it is, (3) the set of users who are assigned the role $r_{1}$ :

1. $\{(u, a, o) \mid \exists r U R A(u, r) \wedge P R A(r, a, o)\}$,

2. $\left\{u \mid \exists s, o, r S U(s, r) \wedge S R(s, r) \wedge \operatorname{Senior} D\left(r, r^{\prime}\right) \wedge P R A\left(r^{\prime}, \mathrm{x}, o\right)\right\}$

3. $\left\{u \mid U R A\left(u, \mathrm{r}_{1}\right)\right\}$

The answer to the last query, on the state given in table 1, is \{Alice, Bob, Charly\}. When I only is queried, we can rely upon any RDBMs to provide the querying mechanism, as the state is stored in extension in relational tables. The technical difficulty is to compute and to query the policy $\mathbf{I}^{\prime}$. As far as Datalog or Datalog ${ }^{C}$ is chosen, $\mathbf{I}^{\prime}$ can be computed quite efficiently. This computation is still valid as long as $\mathbf{I}$ is not modified, thus allowing some caching optimizations techniques. We suggest four approaches to compute the policy $\mathbf{I}^{\prime}$ from a state $\mathbf{I}$ and rules $P$ :

- use recursive queries and advanced features provided by common RDBMS. For instance, Microsoft SQL-Server offers the Common Table Expression system which allows (restricted) recursive queries. From the logical point of view, this kind of feature can be seen as restrictions of DATALOG (e.g., set-based operations are not provided),

- use triggers and stored procedures to compute $\mathbf{I}^{\prime}$ on the fly on each modification of I. This approach can be implemented for simple access control models (when cardinality of $T$ is small enough), but will become hard to maintain if many roles are defined. Indeed it is needed to code specific procedures for each FOL sentence in $P$.

- use a deductive database engine. Several efficient engines have been developed, for instance $\mathrm{DLV}^{3}$ or $\mathrm{XSB}^{4}$. They can compute $\mathbf{I}^{\prime}$ and include recursive queries and simple forms of dependencies. However, expressive dependencies such as TGD are not handled.

\footnotetext{
3 http://www.dbai.tuwien.ac.at/proj/dlv/

4 http://xsb.sourceforge.net/
} 
- develop an external inference engine out of an existing RDBMS while relying on the mechanisms from the RDBMS for access and modification of state. As basic operations are common to both computation of $\mathbf{I}^{\prime}$ and logical inference over $T$ (see next section), we have implemented this approach in a library dedicated to expression and inference of general classes of dependencies.

4.3 Policy completeness and consistency

The validity of an AC policy is checked by verifying, given a model $A C=(\operatorname{Voc}, P, \Sigma)$, whether $\mathbf{I}^{\prime} \models \Sigma$. Unsatisfaction of a set of constraints by a policy can fall into two categories:

- the policy is inconsistent: some CGD or NGD are not satisfied. For instance, a policy is said to be inconsistent if antisymmetry, irreflexivity or exclusion relations are not satisfied,

- the instance is incomplete: some TGD are not satisfied. For instance, a policy is said to be incomplete if some properties of authorization relations or administrative prerequisites are not satisfied.

If a policy is inconsistent or incomplete, administrators have to correct the state. Whenever a policy is inconsistent, deletion of existing facts or value updates should be favored. Whenever a policy is incomplete, addition of facts should be privileged. Examples of possibles corrections of inconsistent or incomplete policies are given in table 6.

\begin{tabular}{llll}
\multicolumn{1}{c}{ Dependency } & Type & Correction in the state \\
\hline \multirow{2}{*}{ property } & antisymmetry & EGD & Deletion of cycles \\
& irreflexivity & NGD & Deletion of edges \\
\cline { 2 - 4 } restrictions & tree hierarchy & EGD & Deletion of ancestors \\
& inverted-tree hierarchy & EGD & Deletion of descendant \\
& lattice hierarchy & TTGD & Addition of edges \\
\cline { 2 - 4 } constraints & prerequisite & CTGD & Addition of requirement \\
& exclusion & NGD & Deletion of assignments \\
& hierarchy/exclusion & NGD & Deletion of assignments \\
\hline
\end{tabular}

Table 6 Examples of inconsistent or incomplete policies corrections

\subsection{Static policy comparison}

Several AC models have introduced dynamic sorts. For instance, in the RBAC family, the unique dynamic sort is subject, the other ones (role, user, action, object, permission) are static, as well as relations between them (e.g., user-role assignment $U R A$, permissionrole assignment $P R A$ ). This specialization can be expressed according to the rights granted to administrators over the state. Sorts and relations in edb can be categorized as follows: 
- static: only administrators can modify this part of the state. Static aspects are stable according to the execution of the system and do not depend on the end-user activity.

- dynamic: administrators are not the only ones having the right to modify this part of the state. For instance, the sort of Subject in RBAC acts on behalf of an user. A $S U$ is created each time a user logged into the system. In AC models involving time or space, these sorts and relations are out of the sovereignty of the administrators.

It is straightforward to define static comparison of policies from the relational paradigm. In section 2.2.1, we have defined three fundamental authorization triples, i.e., Access, Dynamic and Static. Considering the last one, we can compare static restriction of policies by comparing the sets of tuples in Static.

Definition 14 Static comparison of policies. Let be $\mathbf{I}_{\mathbf{1}}$ and $\mathbf{I}_{\mathbf{2}}$ two consistent and complete policies, expressed on two different $\mathrm{AC}$ models $A C_{1}$ et $A C_{2}$.

Let Static $1_{1}$ be the set of static authorization triples of $\mathbf{I}_{\mathbf{1}}$, and Static $\mathrm{C}_{2}$ be the set of static authorization triples of $\mathbf{I}_{\mathbf{2}}$. Static $1 \in \mathbf{I}_{\mathbf{1}}^{\prime} \supseteq \mathbf{I}_{\mathbf{1}}$ et Static ${ }_{2} \in \mathbf{I}_{\mathbf{2}}^{\prime} \supseteq \mathbf{I}_{\mathbf{2}}$.

The policy $\mathbf{I}_{\mathbf{1}}$ is as or more more restrictive than $\mathbf{I}_{\mathbf{2}}$ iff:

$$
\text { Static }_{1} \subseteq \text { Static }_{2}
$$

The policy $\mathbf{I}_{\mathbf{1}}$ is as or more more permissive than $\mathbf{I}_{\mathbf{2}}$ iff:

$$
\text { Static }_{1} \supseteq \text { Static }_{2}
$$

The policies $\mathbf{I}_{\mathbf{1}}$ and $\mathbf{I}_{\mathbf{2}}$ are equivalent iff:

$$
\text { Static }_{1} \subseteq \text { Static }_{2} \text { and Static } 1 ~ \supseteq \text { Static }_{2}
$$

Actually, static sorts constitute the backbone of $\mathrm{AC}$ models. For instance, roles in RBAC, labels in MAC, organizations in OrBAC or tasks in TrBAC are static sorts. Thus, it is worth verifying static enforcement of AC policies, as this can ensure that most robust properties are valid in any state of the policies (Li et al., 2004).

\subsection{Model properties}

Whereas previous subsections have been devoted to policy checking, this section considers $\mathrm{AC}$ models from an abstract perspective, without reference to any particular policy or state. The main problem we address is logical implication, in particular for model simplification purposes. For example, in the case of a new tailored AC model, where many collaborative designers from different sites might be involved, the associated logical theory may become quite large (e.g., hundreds of rules and statements). Thus, for practical purposes, it is necessary to reduce the size of the theory.

Definition 15 Redundancy in an $\mathrm{AC}$ model. Let be $T=P \cup \Sigma$ the logical theory of an $\mathrm{AC}$ model made of rules and constraints. Let be $\sigma \in T$, if $T \backslash\{\sigma\} \models \sigma$ then the dependency $\sigma$ is said redundant, moreover $T \backslash\{\sigma\}$ and $T$ have the same models. 
The authors of Maher and Srivastava (1996) give two bottom-up (also known as forward) chase procedures for solving the implication problem of CTGD: given a set of CTGD $\Sigma$, and a single CTGD $\sigma$ (of the form $\phi \Rightarrow \psi$ ), determine whether in every policy where $\Sigma$ is satisfied, $\sigma$ is also satisfied. If the chase procedures successfully stop, then $\Sigma$ logically implies $\sigma$, stated briefly as $\Sigma \models \sigma$.

The operational nature of proof procedures for CTGD is based on the concept of tuple (a grounded atom, with no variables). The procedures saturates a symbolic database made of tuples by repeated applications of dependencies, to produce a model of the set of dependencies. In a precise sense, this model is the most general possible one, it is a canonical universal one Calì et al. (2008).

Maher and Srivastava's procedures keep the same strategy as the original chase of Beeri and Vardi (1984) extended to deal with constraints. For sake of clarity, original algorithm is given on page 29. Its design is the core of other procedures for dependencies and it is conceptually simpler to understand. It's theoretical complexity has been shown to be exponential (Beeri and Vardi, 1984). The very basic outline of the chase is as follows, input is a set of dependencies $\Sigma$ and a single dependency $\sigma$ :

1. initialization: the chase picks a valuation $\mu$ to hypothesize the existence of some tuples so that the body $\phi$ of $\sigma$ is satisfied,

2. main loop: treats $\Sigma$ as a closure operator generating tuples $\Sigma(\phi)$, that is repeatedly applies dependencies from $\Sigma$ to produce new facts,

3. exit condition: at the end of each loop, the following conditions are checked and three termination cases are possible:

(a) if $\Sigma(\phi)$ contains an inconsistency, return $\Sigma \models \sigma$ vacuously,

(b) if $\Sigma(\phi)$ contains an instance of $\psi$, that is there is a valuation $\nu$ that extends $\mu$ such that $\nu(\psi) \in \Sigma(\phi)$, return $\Sigma \models \sigma$,

(c) if $\Sigma(\phi)$ neither produces new facts nor contains a instance of $\psi$, return $\Sigma \not \models \sigma$.

The CTGD implication problem is semi-decidable: the procedure always halt when the answer is positive, but may run forever if the answer is negative. However, there are some interesting decidability results holding in various subclasses of CTGD such as (Weakly) Guarded-TGD that include existentially quantified variables (Calì et al., 2008). For example, the chase is decidable for TGD having no existentially quantified variable (Beeri and Vardi, 1984).

\subsection{Sample result}

This section illustrates the proposed approach by simplifying integrity properties of mutual exclusion and inheritance defined over a common sort. For this illustation, we use the set of integrity properties for RBAC models defined by Gavrila and Barkley (1998). In the present approach, these properties are modelled by constraints in $\Sigma$. The authors have manually proved that the set of properties in table 7 can be reduced to a smaller set. Using some proof procedures for dependencies, we can simplify their logical theory by eliminating redundancies.

We provide a sample execution trace obtained using a prototype. This prototype, written in $\mathrm{C}++$, can handle the CTGD as well as its subclasses. It relies on an external constraint solver over reals to handle constraints. Three different chases have been implemented : (Beeri and Vardi, 1984), (Maher and Srivastava, 1996) and (Coulondre, 2003). Given a logical formalization of an AC written using dependencies, the prototype 
Description

$\sigma_{1} \quad$ any two roles assigned for a same user are not in separation of dutie $U R A\left(U s e r\right.$, Role $\left._{1}\right), U R A\left(U\right.$ ser Role $\left._{2}\right)$, SoD $\left(\right.$ Role $_{1}$, Role $\left._{2}\right) \Rightarrow \perp$

$\sigma_{2} \quad$ no role is mutually exclusive with itself $\operatorname{SoD}($ Role, Role $) \Rightarrow \perp$

$\sigma_{3} \quad$ mutual exclusion is symmetric SoD $\left(\right.$ Role $_{1}$, Role $\left._{2}\right) \Rightarrow \operatorname{SoD}\left(\right.$ Role $_{2}$, Role $\left._{1}\right)$

$\sigma_{4} \quad$ any two roles in ssd do not inherit one another Senior $\left(\right.$ Role $_{1}$, Role $\left._{2}\right), \operatorname{SoD}\left(\right.$ Role $_{1}$, Role $\left._{2}\right) \Rightarrow \perp$

$\sigma_{5}$ there is no role inheriting two roles in ssd SoD $\left(\right.$ Role $_{1}$, Role $\left._{2}\right)$, Senior (Senior, Role 1$)$, Senior $($ Senior, Role 2$) \Rightarrow \perp$

$\sigma_{6} \quad$ If a role inherits another role and that role is in SSD with a third one, then the inheriting role is in SSD with the third one. Senior $\left(\right.$ Senior, Role $\left.{ }_{1}\right), \operatorname{SoD}\left(\right.$ Role $_{1}$, Role $\left._{2}\right) \Rightarrow \operatorname{SoD}($ Senior, Role 2$)$.

Table 7 Logical characterization of RBAC constraints (Gavrila and Barkley, 1998)

is used to simplify the model, to compute the policy for a given state, to check the consistency of the policy and to answer administrative queries. Static comparison of policies have not been implemented yet.

The following trace is the result of the execution of the CTGD chase of Maher and Srivastava (1996) implemented in the prototype. Initially, the dependency base is loaded with $\sigma_{2}, \sigma_{3}$ and $\sigma_{6}$ of table 7 . The goal is to prove that $\left\{\sigma_{2}, \sigma_{3}, \sigma_{6}\right\}=\sigma_{5}$. The following trace is a formal proof of this entailment.

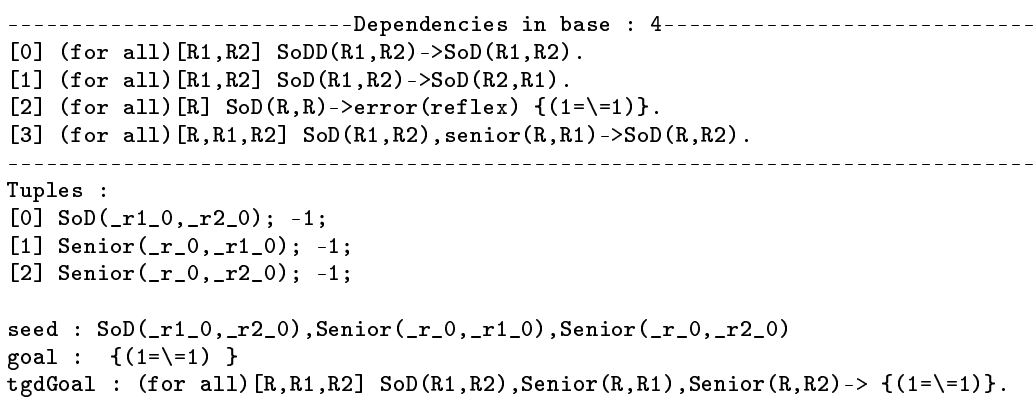

Hypothesize three tuples $\operatorname{SoD}\left(\mathrm{r}_{1}, \mathrm{r}_{2}\right)$, Senior $\left(\mathrm{r}_{0}, \mathrm{r}_{1}\right)$ and $\operatorname{Senior}\left(\mathrm{r}_{0}, \mathrm{r}_{2}\right)$ from the body of $\sigma_{5}$.

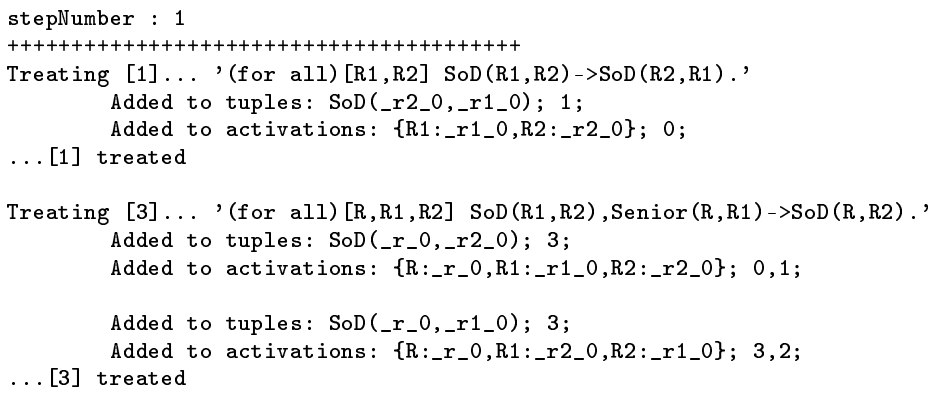


With $\sigma_{3}$ (sentence [1], mutual exclusion is symetric), $\operatorname{SoD}\left(\mathrm{r}_{2}, \mathrm{r}_{1}\right)$ is derived. Next, applying $\sigma_{6}$ (sentence [3], exclusion is propagated through inheritance) twice, $S o D\left(\mathrm{r}_{0}, \mathrm{r}_{2}\right)$ et $\operatorname{SoD}\left(\mathrm{r}_{0}, \mathrm{r}_{1}\right)$ is derived.

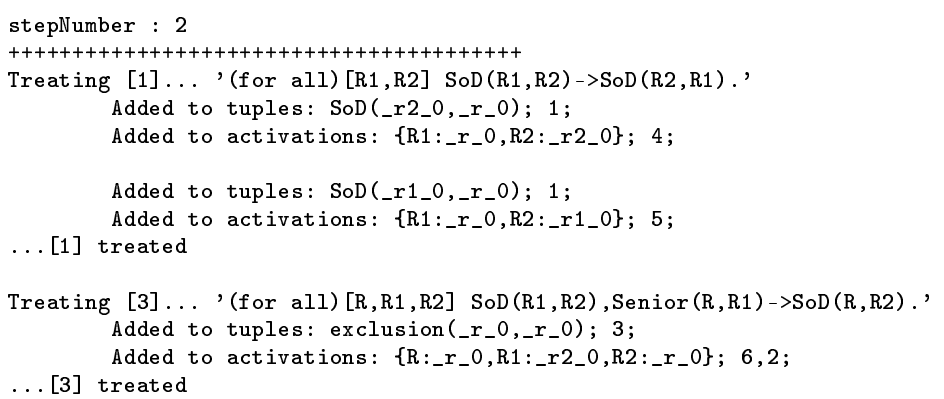

Next, using $\sigma_{3}$ (sentence [1]), $S o D\left(\mathrm{r}_{2}, \mathrm{r}_{0}\right)$ and $S o D\left(\mathrm{r}_{1}, \mathrm{r}_{0}\right)$ are deduced. Thus, by $\sigma_{6}$ (sentence [3]), $S o D\left(r_{0}, r_{0}\right)$ is derived.

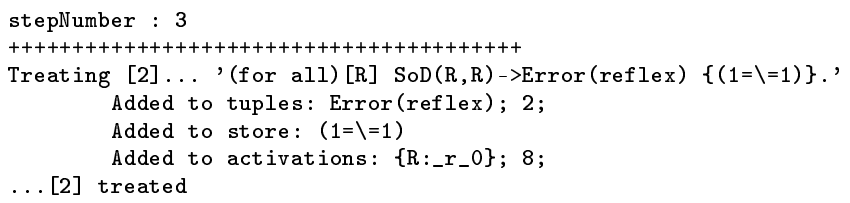

Finally, applying $\sigma_{2}$ (sentence [2], exclusion is irreflexive), an antilogy is derived. Thus, the chase procedure proved that $\left\{\sigma_{2}, \sigma_{3}, \sigma_{6}\right\} \models \sigma_{5}$. The prototype can be used to derive other sample theorem from the dependencies of table 7 . Let be $\Sigma$ the six dependencies shown in the table. The chase procedures can prove that $\Sigma \backslash\left\{\sigma_{4}\right\} \models \sigma_{4}$, $\Sigma \backslash\left\{\sigma_{5}\right\} \models \sigma_{5}$ and that $\Sigma \backslash\left\{\sigma_{4}, \sigma_{5}\right\} \models \sigma_{4}, \sigma_{5}$.

This example illustrates the utility of the proposed framework. Given an AC model $A C=(V o c, P, \Sigma)$, an automated proof of non-trivial properties can be provided. For instance, besides the above obtained theorems, we have been able to derive the following results:

- read and write access over an objet in Mandatory Access Control (MAC) are granted to a subject iff the subject's clearance level is equal to the object's confidentiality level (Sandhu, 1993),

- a root role which inherits all other ones cannot exist in an RBAC policy where two roles are mutually exclusive (Benantar, 2006),

- dynamic authorizations are a subset of static authorizations in RBAC policies (Ferraiolo et al., 2003). 


\section{Related work}

\subsection{General comparison}

Our work is closely related to (Barker and Stuckey, 2003) which expresses RBAC models in constraint logic programming, to (Halpern and Weissman, 2003) which identifies a subset of Fol for AC models, to (Bertino et al., 2003) which describes a C-DATALOG framework for representing AC models, to (Jajodia et al., 2001) which defines a flexible access control framework, to ( $\mathrm{Li}$ and Mitchell, 2003) which uses DATALOG ${ }^{C}$ and (Miège, $2005)$ which uses the DATALOG $\urcorner$ for defining the OrBAC model. Our main goals were to provide a framework for $\mathrm{AC}$ which:

- makes a clear separation between policies and models and has a clear semantics,

- is expressive enough to capture and generalize roperties of AC models,

- treats policy constraints as first-class citizens,

- provides readable algorithm execution traces for designers and administrators.

The background we have settled in section 2 is based on DATALOG ${ }^{C}$. This logical framework has been often treated as a middle-ground formalism to which many other logical based frameworks can be reduced, for instance in the work of (DeTreville, 2002; Jim, 2001; Bertino et al., 2003; Li and Mitchell, 2003). Moreover, Datalog ${ }^{C}$ has shown to be able to capture several AC models, and various extensions of RBAC model (Joshi et al., 2005; Damiani et al., 2007). As the present framework subsumes DATALOG ${ }^{C}$, it can basically capture these models.

The main difference with the above previous work is the fruitful use of data dependencies as a unifying logical framework which encompasses both traditional AC rules and integrity constraints. Thus, constraints are expressed in the very same model, and not expressed in an independent and different framework. As constraints are integrity requirements of policies, we argue that integrating them in the model as soon and as tightly as possible is a step towards ensuring AC robustness.

We rely on some known results for data dependencies, in order to provide wellfounded tools for reasoning on policies. An interesting feature of these proof procedures is that they do not require any prior translation of FOL formulae of rules $P$ and integrity constraints $\Sigma$ (e.g., by means of Skolemization or rewriting rules). This property leads to native clear traces of automated proofs, as given in section 4.6. This greatly enhances the readability of inference results for design and maintenance purposes. Finally, by means of the prototype, we have been able to re-prove in an automated way some interesting results found in the literature.

\subsection{Frameworks built upon Datalog}

(Bertino et al., 2003) and ( $\mathrm{Li}$ and Mitchell, 2003) have used the Datalog framework, respectively C-DATALOG which allows object-oriented definitions, and DATA${ }_{\text {LOG }}{ }^{C}$ which includes constraints. These frameworks are able to capture extended RBAC models such as Temporal-RBAC (Bertino et al., 2001). The basic components of the present proposition (sections 2 and 2.2) reuse and extend some of their formal models. (Li et al., 2003), (Jim, 2001), and (DeTreville, 2002) have extended Datalog with specific constructions which are reduced into standard DATALOG formulae. However, we have not used rewriting procedures which may cloud the debugging steps and puzzle administrators. 
5.3 Other frameworks built upon logic

(Jajodia et al., 2001), (Barker and Stuckey, 2003) and (Halpern and Weissman, 2003) describe logical programs able to express general AC models. These propositions address the problems arising with the use of closed policies (in which access is by default denied and authorizations are only positive), open policies (access is granted by default) or hybrid policies (authorizations and denial can be both explicitly defined). Miège (2005) use DATALOG $\urcorner$ to deal with negative authorization (prohibition). These logical frameworks allow a permissive use of negation in formal sentences, whereas we chose to favor existential quantifiers. However, our framework is able to support a restrictive use of negation by means of NGD for instance. Our framework captures complex integrity requirements considered as fundamental in RBAC, which are not expressible in other ones. Moreover, we provide an effective procedure to automatize administrative operations.

Our framework is tightly linked to the database area and makes a clear distinction between policies and models. This separation, advocated by (Li et al., 2007), is not explicit in the work of Barker and Stuckey (2003) and Halpern and Weissman (2003), as well as in the logic programming paradigm on a more general point of view. Finally, we have reused and extended some definitions of (Ferraiolo et al., 2003) (properties of RBAC) and results of (Gavrila and Barkley, 1998) (consistency of RBAC databases) which were not integrated into other frameworks.

\subsection{Access control constraints}

Constraints have received much attention in AC models. RBAC is argueably one of their most prominent representatives for which many kinds of constraints have been proposed (Gligor et al., 1998; Ahn and Sandhu, 1999; Crampton, 2003). These suggestions either define and categorize new kinds of constraints (e.g., the variations on mutual exclusion outlined in section 3.4) or proposed specification languages for constraints. Most of these constraints are defined as a way to enforce separation of duties, and are variants of mutual exclusion.

The algebra of Li and Wang (2008) for specifying constraints encompasses these approaches but consider constraints as high-level organization requirements. Our approach is quite different, in the sense that constraints are defined closer to the model at a lower level. Thus, we can capture intrinsic properties (e.g., constraints on authorization triples, prerequisites) as well as general integrity requirements (e.g., states well-foundedness, algebraic properties of relations) which are usually taken into account separately.

\section{Discussion}

This section discusses the main choices we made. We divide this section into the AC structure design choices and the FOL fragment choice. 
6.1 Design and administration

By drawing a distinction between an AC model and an AC policy instanciated from a model, we can define two AC-related activities:

- model design is the task of defining the AC model. The model designer sets up the sorts and relations of the model $(V o c)$, its rules $(P)$ and the properties that must be enforced $(\Sigma)$. For instance, a model designer may define that tasks and roles are used to structure policies. As an analogy in the database field, the model designer counterpart is the database administrator, who defines tables $(V o c)$, views $(P)$ and keys between tables $(\Sigma)$.

- policy administration is the task of defining the state $\mathbf{I}$. The policy administrator sets up the facts of an AC model instance, but can not define $\mathbf{I}^{\prime}$ directly because it is automatically derived from $\mathbf{I}$ using $P$. According to the above example, a policy administrator sets that physician and surgeon are roles, and is responsible for assigning these roles to individuals. As an analogy in the database field, the policy administrator counterpart is an end-user, whose job is to query and modify the policy, but who is not able to modify the schema.

\subsection{Model structure}

In section 2, we introduced the structure of $A C=(\operatorname{Voc}, P, \Sigma)$ made of three components and we defined the major design choices we have made:

- AC models are defined as logical theories while being narrowed to decidable fragments without negation, disjunction nor function symbols in $P$,

- the vocabulary is partitioned into sorts and relations in a many-sorted Fol paradigm,

- rules $(P)$ and constraints $(\Sigma)$ are distinguished,

- the difference between a state $\mathbf{I}$ and a policy $\mathbf{I}^{\prime}$ is logically made explicit.

One may argue that these choices are somewhat restrictive. The administrator's tasks are limited to extensive policies management, i.e., to tuple management in the AC state. It might be possible to allow them to expressed their own formulae in $P$ or $\Sigma$. However, we think this is not desirable, as this would blur the responsabilities between designers and maintainers and would introduce higher policy checking complexity. In such an approach, the stability of the AC models would not be guaranteed through the policies lifetime, in particular consistency and interoperability of updated models.

\subsection{Fragments of logic for access control}

The logical fragment of FOL we used to define $P$ and $\Sigma$ is quite restrictive: negation, disjunction and function symbols are not allowed. The main argument is that we obtain a unique and computable model of $P$. Computability is necessary because the reference monitor has to answer each access request. Uniqueness for integrity checking purposes is required, in order to avoid checking the satisfaction of $\Sigma$ over multiple models of $P$. Moreover, uniqueness may ease the understanding of intensive policies by administrators.

Furthermore, we chose to have a more expressive framework for constraints than for rules, by favoring existential quantification over negation. The main goal is to be able 
to model some of the most important constraints commonly identified for AC models, which cannot be expressed in DATALOG models. Even with a quite simple AC model (for instance, with a few sorts, relations and principles), most of the properties given in section 3 require existentially quantified variables (e.g., sessions integrity properties, restricted hierarchies, authorizations properties and administrative prerequisites).

\section{Conclusions and perspectives}

In this paper, we presented a logical framework $A C=(V o c, P, \Sigma)$ for $\mathrm{AC}$ models, which relies on three abstract components. The key idea is to express and handle AC models, policies and constraints in an homogeneous way within the very same logic background by means of database integrity theory (not to deploy AC policies in databases). We then showed how to use well-founded procedures in order to enforce and check constraints, and to provide a formal trace of inferences. The proposed framework is not universal because of the choice we made to focus on access control constraints and to treat them as first-class citizens. It however allows for expressing several classical models and extensions found in the literature. It also takes into account some major recommendations that had been previously addressed to RBAC models. Moreover, the expressivity of general classes of dependencies allows capturing most of the AC model properties considered as fundamental.

We envision several extensions and perspectives:

- extension of the FOL subclasses, to capture new properties of models. However, algorithms decidablity and tractability should be taken into account. As an example, it may be interesting to use some decidable subclass of TGD by imposing some restrictions on existentially quantified variables, as it is the case for negation in stratified-DATALOG for instance,

- explore automated maintenance of databases (Chomicki and Marcinkowski, 2005), and data integration (Fagin, 2006) to fix non-consistent policies. Data integration may be a fruitful perspective for policies creation expressed in different models ( $\mathrm{Li}$ et al., 2009),

- broadening the scope of policies. Indeed, it would be valuable to model administrative policies, which define administrator rights over AC policies. This could be useful for instance when a huge policy requires several administrators, each of them being allowed to handle only a part of the policy.

- another emerging topic is usage control and privacy protection. The basic components and definitions we presented can be used to define next generation AC models and policies, as it has been done with RBAC models (Ni et al., 2009).

\section{References}

Serge Abiteboul, Richard Hull, and Victor Vianu. Foundations of Databases. AddisonWesley, Boston, 1995. ISBN 0-201-53771-0.

Gail-Joon Ahn and Ravi S. Sandhu. The RSL99 language for role-based separation of duty constraints. In RBAC'99: 4th ACM workshop on Role-based access control, pages 43-54, New York, 1999. ACM Press. ISBN 1-58113-180-1. doi: http://doi. acm.org/10.1145/319171.319176. 
Steve Barker and Peter J. Stuckey. Flexible access control policy specification with constraint logic programming. ACM Transactions on Information 8 S System Security, 6(4):501-546, 2003.

Catriel Beeri and Moshe Y. Vardi. A proof procedure for data dependencies. Journal of the ACM, 31(4):718-741, 1984.

Messaoud Benantar, editor. Access Control Systems - Security, Identity Management and Trust Models. Springer-Verlag, 2006.

Elisa Bertino, Piero A. Bonatti, and Elena Ferrari. TRBAC: A temporal role-based access control model. ACM Transactions on Information \& System Security, 4(3): 191-233, 2001

Elisa Bertino, Barbara Catania, Elena Ferrari, and Paolo Perlasca. A logical framework for reasoning about access control models. ACM Transactions on Information $\mathcal{G}$ System Security, 6(1):71-127, 2003.

Andrea Calì, Georg Gottlob, and Michael Kifer. Taming the infinite chase: Query answering under expressive relational constraints. In Gerhard Brewka and Jérôme Lang, editors, $K R$, pages 70-80. AAAI Press, 2008. ISBN 978-1-57735-384-3.

Jan Chomicki and Jerzy Marcinkowski. Minimal-change integrity maintenance using tuple deletions. Information \& Computation, 197(1-2):90-121, 2005.

Stéphane Coulondre. A top-down proof procedure for generalized data dependencies. Acta Informatica, 39(1):1-29, 2003.

Jason Crampton. Specifying and enforcing constraints in role-based access control. In SACMAT'03: 8th ACM Symposium on Access Control Models and Technologies, pages 43-50. ACM Press, 2003. ISBN 1-58113-681-1. doi: http://doi.acm.org/10. $1145 / 775412.775419$.

Maria Luisa Damiani, Elisa Bertino, Barbara Catania, and Paolo Perlasca. GEORBAC: A spatially aware rbac. ACM Transactions on Information $\&$ System Security, 10(1), 2007.

John DeTreville. Binder, a logic-based security language. In SP'02: IEEE Symposium on Security and Privacy, page 105, Washington, 2002. IEEE Computer Society. ISBN 0-7695-1543-6.

Ronald Fagin. Inverting schema mappings. In Stijn Vansummeren, editor, PODS'06: 25th ACM SIGACT-SIGMOD-SIGART Symposiumon Principles of Database Systems, Chicago, Illinois, pages 50-59. ACM Press, 2006. ISBN 1-59593-318-2.

David F. Ferraiolo, Richard D. Kuhn, and Ramaswamy Chandramouli. Role-Based Access Control. Artech House Publishers, 2003. ISBN 1-58053-370-1.

Jean H. Gallier. Logic for Computer Science: Foundations of Automatic Theorem Proving. Revised on-line version 2003. Harper \& Row, 1986. ISBN 0-06-042225-4. http://www.cis.upenn.edu/ jean/gbooks/logic.html.

Serban I. Gavrila and John F. Barkley. Formal specification for role based access control user/role and role/role relationship management. In RBAC'98: 3rd ACM workshop on Role-based access control, pages 81-90, 1998.

Virgil D. Gligor, Serban I. Gavrila, and David F. Ferraiolo. On the formal definition of separation-of-duty policies and their composition. In 1998 Symposium on Security and Privacy, pages 172-183, Oakland, California, 1998. IEEE Computer Society Press.

Joseph Y. Halpern and Vicky Weissman. Using first-order logic to reason about policies. In CSFW'03: 16th IEEE Computer Security Foundations Workshop, Pacific Grove, California, pages 187-201. IEEE Computer Society, 2003. ISBN 0-7695-1927-X. 
Trent Jaeger and Jonathon E. Tidswell. Practical safety in flexible access control models. ACM Trans. Inf. Syst. Secur., 4(2):158-190, 2001. ISSN 1094-9224. doi: http://doi.acm.org/10.1145/501963.501966.

Sushil Jajodia, Pierangela Samarati, Maria Luisa Sapino, and V. S. Subrahmanian. Flexible support for multiple access control policies. ACM Trans. Database Syst., 26:214-260, June 2001. ISSN 0362-5915. doi: http://doi.acm.org/10.1145/383891. 383894. URL http://doi.acm.org/10.1145/383891.383894.

Trevor Jim. SD3: A trust management system with certified evaluation. In IEEE Symposium on Security and Privacy, pages 106-115, 2001.

James Joshi, Elisa Bertino, Usman Latif, and Arif Ghafoor. A generalized temporal role-based access control model. IEEE Transactions on Knowledge \& 3 Data Engineering, 17(1):4-23, 2005.

Richard D. Kuhn. Mutual exclusion of roles as a means of implementing separation of duty in role-based access control systems. In RBAC'97: 2nd ACM workshop on Rolebased access control, pages 23-30, New York, 1997. ACM Press. ISBN 0-89791-985-8. doi: http://doi.acm.org/10.1145/266741.266749.

Butler W. Lampson. Protection. ACM SIGOPS Operating Systems Review, 8(1):18-24, 1974. ISSN 0163-5980. doi: http://doi.acm.org/10.1145/775265.775268.

Ninghui Li and John C. Mitchell. DATALOG with constraints: A foundation for trust management languages. In Verónica Dahl and Philip Wadler, editors, $P A D L$ '03: 5th International Symposium on Practical Aspects of Declarative Languages, New Orleans, volume 2562 of Lecture Notes in Computer Science, pages 58-73. SpringerVerlag, 2003. ISBN 3-540-00389-4.

Ninghui Li and Qihua Wang. Beyond separation of duty: An algebra for specifying high-level security policies. J. ACM, 55(3), 2008.

Ninghui Li, Benjamin N. Grosof, and Joan Feigenbaum. Delegation logic: A logic-based approach to distributed authorization. ACM Transactions on Information \& System Security, 6(1):128-171, 2003.

Ninghui Li, Ziad Bizri, and Mahesh V. Tripunitara. On mutually-exclusive roles and separation of duty. In CCS'04: 11th ACM conference on Computer and communications security, pages 42-51, New York, 2004. ACM Press. ISBN 1-58113-961-6. doi: http://doi.acm.org/10.1145/1030083.1030091.

Ninghui Li, Ji-Won Byun, and Elisa Bertino. A critique of the ANSI standard on rolebased access control. IEEE Security and Privacy, 5(6):41-49, 2007. ISSN 1540-7993. doi: http://doi.ieeecomputersociety.org/10.1109/MSP.2007.158.

Ninghui Li, Qihua Wang, Wahbeh Qardaji, Elisa Bertino, Prathima Rao, Jorge Lobo, and Dan Lin. Access control policy combining: theory meets practice. In SACMAT '09: Proceedings of the 14th ACM symposium on Access control models and technologies, pages 135-144. ACM, 2009. ISBN 978-1-60558-537-6. doi: http://doi.acm.org/10.1145/1542207.1542229.

Michael J. Maher and Divesh Srivastava. Chasing constrained tuple-generating dependencies. In Richard Hull, editor, PODS'96: 15th ACM SIGACT-SIGMOD-SIGART Symposium on Principles of Database Systems, Montreal, Canada, pages 128-138. ACM Press, 1996. ISBN 0-89791-781-2.

Alexandre Miège. Dénition d'un environnement formel d'expression de politiques de sécurité : modèle Or-BAC et extensions. PhD thesis, Ecole Nationale Supérieure des Télécommunications,Paris, 2005.

Qun Ni, Elisa Bertino, and Jorge Lobo. Privacy-aware RBAC - leveraging RBAC for privacy. IEEE Security and Privacy, to appear, 2009. 
Ravi S. Sandhu. Lattice-based access control models. Computer, 26(11):9-19, 1993. ISSN 0018-9162. doi: http://doi.ieeecomputersociety.org/10.1109/2.241422.

Ravi S. Sandhu, Edward J. Coyne, Hal L. Feinstein, and CharlesE. Youman. Role-based access control models. IEEE Computer, 29(2):38-47, 1996.

Roshan K. Thomas. Team-based access control (tmac): a primitive for applying rolebased access controls in collaborative environments. In RBAC'97: 2nd ACM workshop on Role-based access control, pages 13-19, New York, 1997. ACM Press. ISBN 0-89791-985-8. doi: http://doi.acm.org/10.1145/266741.266748.

Roshan K. Thomas and Ravi S. Sandhu. Task-based authorization controls (TBAC): A family of models for active and enterprise-oriented autorization management. In Tsau Young Lin and Shelly Qian, editors, IFIP'98: 11th International Conference on Database Security, Lake Tahoe, California, volume 113 of IFIP Conference Proceedings, pages 166-181. Chapman \& Hall, 1997. ISBN 0-412-82090-0.

Jacques Wainer, Paulo Barthelmess, and Akhil Kumar. W-RBAC - a workflow security model incorporating controlled overriding of constraints. International Journal of Cooperative Information Systems, 12(4):455-485, 2003.

Jacques Wainer, Akhil Kumar, and Paulo Barthelmess. DW-RBAC: A formal security model of delegation and revocation in workflow systems. Information Systems, 32 (3):365-384, 2007. 


\section{A Chase algorithm}

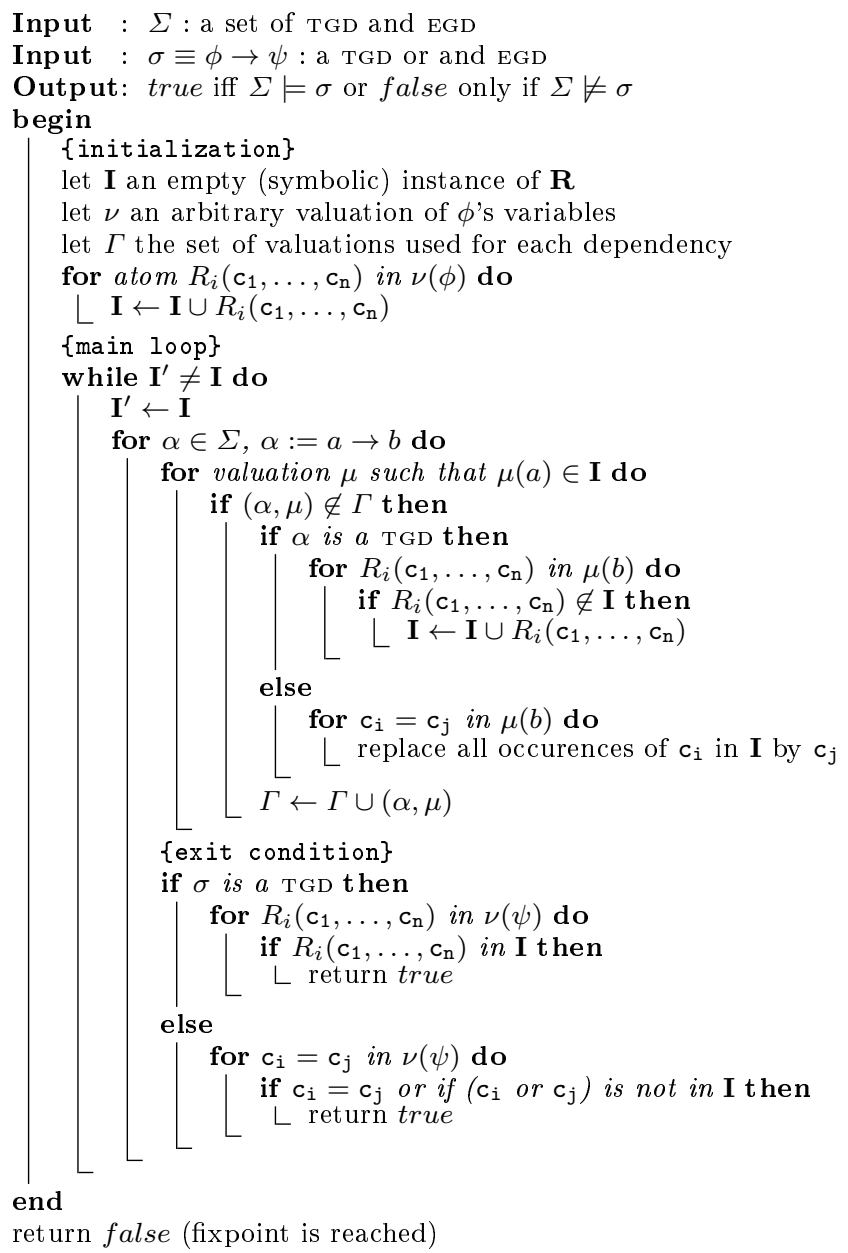

Algorithm 1: Chase algorithm from Beeri and Vardi (1984) 